\title{
Analysis of Problems in Wind Power Generation Based on Artificial Intelligence
}

\author{
Jia-Jun ZHANG ${ }^{1, a}$, Xin-Yan ZHANG ${ }^{1,2, b, *}$, Liang GAO $^{1, c}$ and Tao TONG ${ }^{1}$, \\ ${ }^{1}$ College of Electric engineering, Xinjiang University, China \\ ${ }^{2}$ Engineering Research Center of Education Ministry for Renewable Energy Power Generation and \\ Grid Technology, Xinjiang University, China \\ a1624236670@qq.com, bxjcxzxy@126.com, c1793346200@qq.com
}

${ }^{\star}$ Corresponding author

Keywords: New energy utilization, Artificial intelligence, Wind power generation, Fault diagnosis.

\begin{abstract}
Wind energy is widely used as clean, renewable and mature new energy. However, the inhomogeneity and non-steady state of the operating environment of the wind turbine lead to the randomness of the load, which will cause the fluctuation of the voltage and frequency of the power grid, and affect the power quality of the power grid; the wind turbine will also have various faults, which will cause the unit to stop and reduce the utilization rate of the unit. Artificial intelligence technology can diagnose and predict wind power for wind turbines, so that new energy can be better complemented with traditional hydro thermal power. Finally, an example of fault diagnosis of daily monitoring data of doubly fed induction generator in Dabancheng, Xinjiang is given to demonstrate the application of artificial intelligence technology in wind power generation.
\end{abstract}

\section{Introduction}

At present, the increasing depletion of fossil energy and the increasingly serious environmental pollution problems, the development and utilization of new energy, the protection of energy supply and energy security, the reduction of energy consumption, the reduction of environmental pollution, and the response to climate change have become the consensus of the world's [1]. As a new pollution-free, renewable and mature energy, wind energy has been developed and utilized all over the world. However, wind energy has the characteristics of intermittent and time-varying [2], which makes the wind power generation influence the original dynamic balance of the power grid and reduce the power quality of the power grid, which makes the development of the new energy limited.

With the development of big data, cloud computing and artificial intelligence, there have been fundamental changes in all walks of life. The power industry is also affected. In recent years, the application of Expert Systems (ES), Artificial Neural Network (ANN), Fuzzy Theory (FT), Genetic Algorithm (GA) and Multiple Agent System (MAS) technology have made great achievements in the power system, which has made great achievements in the power system.

\section{Large Data of Power System}

Large data refers to the collection of data that cannot be captured, managed and processed by conventional software tools in a certain time range. It is a massive, high growth rate and diversified information asset that needs a new processing mode with stronger decision-making power, insight, and process optimization.

Power big data refers to the large number of data collected by power grid in power generation, distribution, transmission, marketing and management, etc. [4]. Through the analysis of large power data, it can improve the lean management level of the smart grid, make a more scientific production plan, optimize the energy transport scheduling, and establish a more accurate user power consumption behavior model. 
The flow of power big data from collection to analysis and calculation is shown in Fig. 1. First, a large number of sensors and smart meters are used to collect data periodically. In order to eliminate errors in data acquisition process and alleviate the access pressure of cloud storage system, the data collected first go to the front processor for pre-processing. Parallel computing environment access cloud storage system, according to the business logic of the data to carry out complex analysis and calculation, and write the results to the cloud storage system. The online query system obtains data that meets user request from the cloud storage system and returns to user [4].

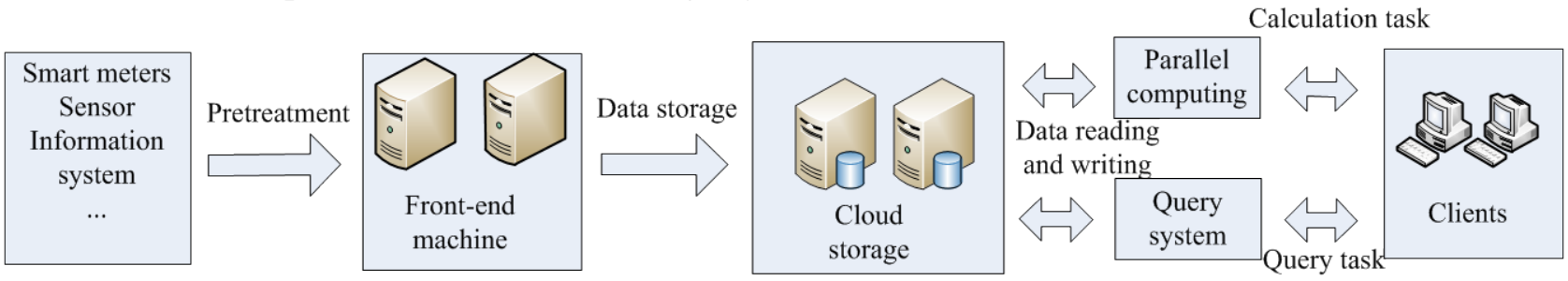

Fig. 1 The flow of power big data

\section{Application of Artificial Intelligence in New Energy Power Generation}

Wind power prediction. Due to the influence of weather, season and other factors, the wind flow has the characteristics of space inhomogeneity, time unsteady and high turbulence intensity. The output of wind turbine is mainly determined by the wind speed, so the power output of the wind farm is fluctuating $[5,6]$. When the large-scale wind power is connected to the grid, the instability will cause the fluctuation of the grid voltage and frequency, which will affect the power quality of the power grid. In order to eliminate the adverse effects, the Power Grid Corp needs to increase the extra rotating spare capacity and increase the operation cost of the power grid, so it is particularly important to predict the wind speed in advance. Artificial neural network is an important part of artificial intelligence; artificial neural network algorithm is to simulate human thinking, training and learning through a large amount of historical data, and forecast for future data. The trained neural network has strong accuracy, fault tolerance and robustness [7].

Fault diagnosis. With the increase of operating time, the wind turbine blade, generator, gear box, and control system may have various kinds of fault [8], which will cause the unit to stop, which will reduce the utilization rate of the unit. According to incomplete statistics, the average utilization rate of wind turbines in wind farms in China is generally lower than 95\%, except that the wind power access system is not available, and the high failure rate of the wind turbine is a major factor [9]. Artificial intelligence technology can detect faults in the early stage of failure, and deal with them in time to reduce the damage. By extracting the data features of the sensor, we can use the support vector machine and other algorithms to classify and identify the parts that have occurred. It is convenient to replace the fault parts in maintenance, greatly reducing the downtime of the small wind turbine and increasing the economic benefit of the wind power field.

\section{An Example Analysis on Fault Diagnosis of Wind Turbine}

Equipment fault diagnosis is actually the process of pattern recognition, which includes the feature extraction process and the fault recognition process [10]. It is intuitively that the method of fault diagnosis based on pattern recognition is to classify the running state and fault of a system through a pattern recognition system, including signal acquisition, feature value extraction and classification recognition, as shown in Fig. 2.

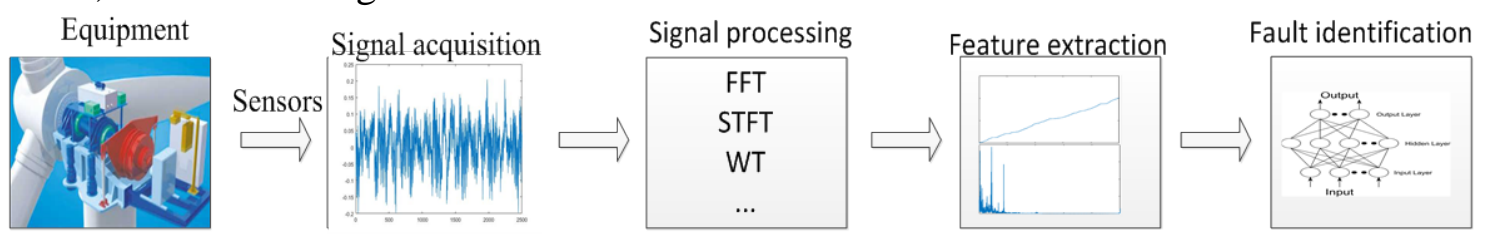

Fig. 2 Process of pattern recognition 


\section{Signal Acquisition}

The daily monitoring data of Xinjiang Dabancheng doubly fed wind turbine in the second half of 2017 was statistically analyzed, and 40 groups of vibration data samples were selected from four states, including normal operation, spindle eccentricity, bearing wear and stator winding interturn short circuit, of which 28 groups were training samples and 12 groups were tested. As shown in Fig. 3 , the vibration data of the wind turbine in four states:

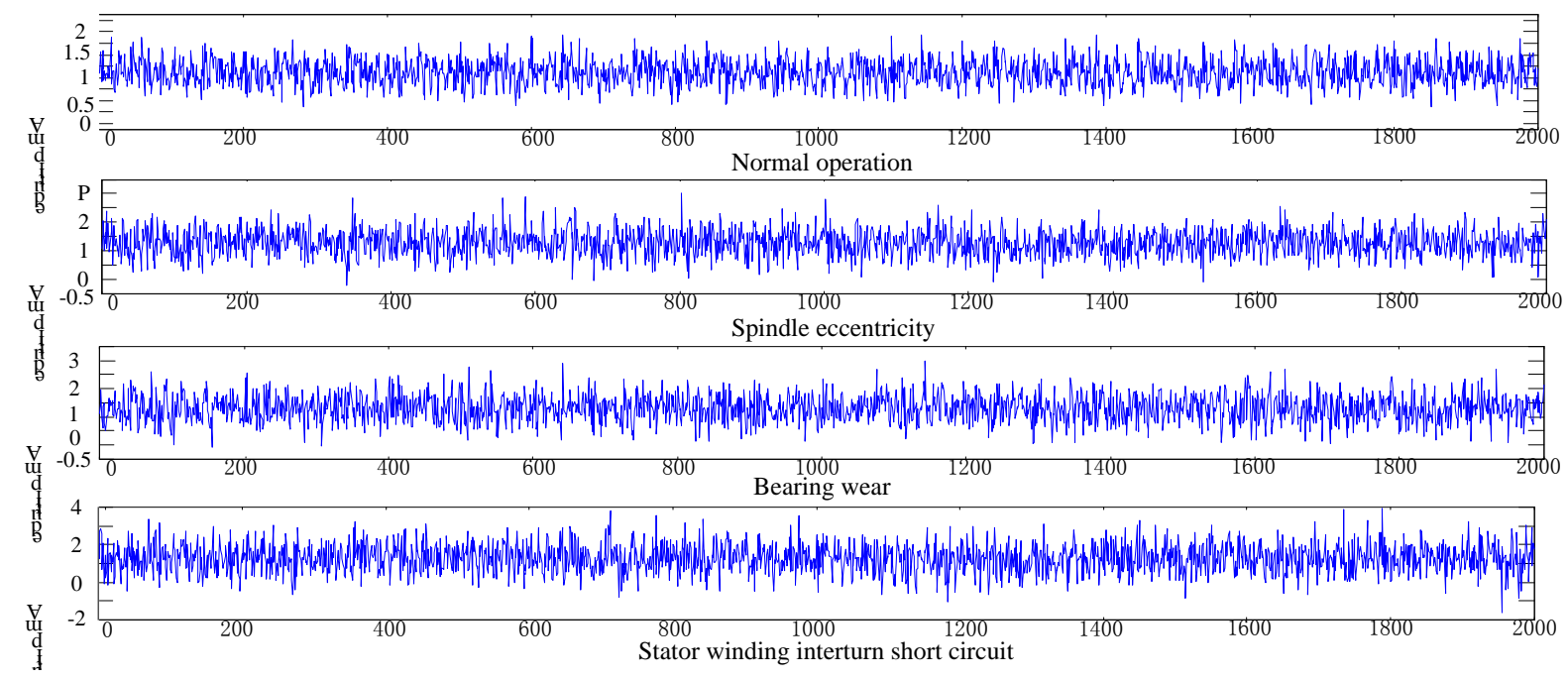

Fig. 3 Vibration data of four states

\section{Signal Processing and Fault Feature Extraction}

The 160 sets of sample data are extracted and the fault features are extracted. The wavelet packet decomposition and reconstruction of the four state vibration signals are selected by the wavelet basis function $\mathrm{db} 8$, and the wavelet packet decomposition and reconstruction coefficient diagram of the normal state of the wind generator is shown as Fig. 4. The signal values are reconstructed by using the 8 nodes of third layers decomposed by wavelet packet, and the energy values are obtained. The normalization is used as the vibration eigenvector of the vibration signal.

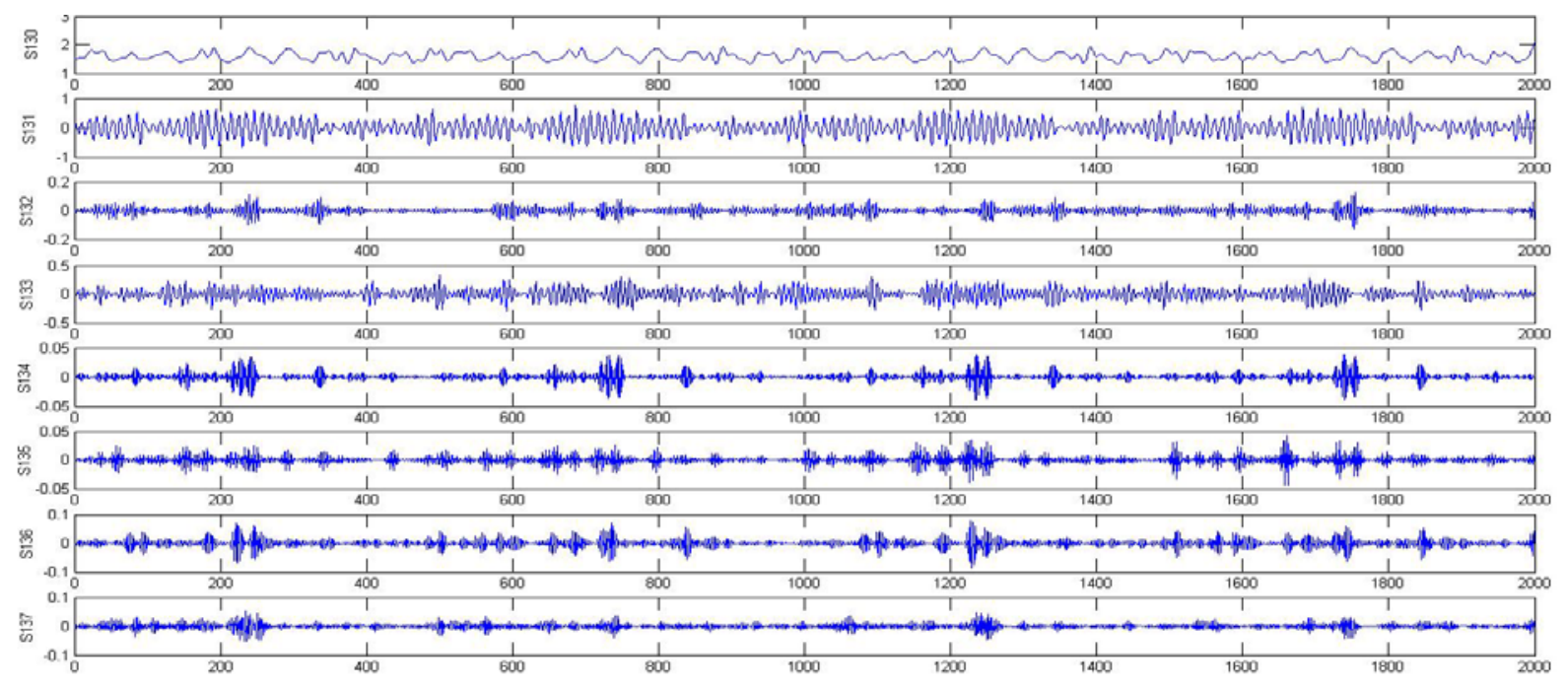

Fig. 4 the Reconstructed Coefficient Map of 8 Frequency Bands after Wavelet Packet Decomposition

The energy values of the reconstructed signal coefficients of the 8 nodes are calculated and normalized as F1= [E1, E2, E3, E4, E5, E6, E7, E8] of the vibration signal vibration characteristics. The eigenvectors of vibration signals of wind turbines are shown in Table 1. 
Table 1 Vibration signal normalized eigenvectors in each operating state

\begin{tabular}{|c|c|c|c|c|c|c|c|c|c|}
\hline \multirow{2}{*}{ No. } & \multirow{2}{*}{ Sample type } & \multicolumn{8}{|c|}{ Fault features } \\
\hline & & $E_{1}$ & $E_{2}$ & $E_{3}$ & $E_{4}$ & $E_{5}$ & $E_{6}$ & $E_{7}$ & $E_{8}$ \\
\hline 1 & \multirow{2}{*}{ normal status } & 0.6237 & 0.1028 & 0.0896 & 0.1148 & 0.0023 & 0.0182 & 0.047 & 0.0016 \\
\hline 2 & & 0.5942 & 0.1273 & 0.1095 & 0.0956 & 0.0116 & 0.0094 & 0.0035 & 0.0489 \\
\hline 3 & \multirow{2}{*}{$\begin{array}{c}\text { Spindle } \\
\text { eccentricity }\end{array}$} & 0.5139 & 0.0405 & 0.2189 & 0.1176 & 0.0892 & 0.0062 & 0.0012 & 0.0125 \\
\hline 4 & & 0.4899 & 0.0892 & 0.1965 & 0.1237 & 0.0769 & 0.0059 & 0.0015 & 0.0164 \\
\hline 5 & \multirow{2}{*}{ Bearing wear } & 0.2407 & 0.1969 & 0.1219 & 0.1614 & 0.0437 & 0.0696 & 0.0852 & 0.0806 \\
\hline 6 & & 0.2309 & 0.2205 & 0.1231 & 0.1403 & 0.0485 & 0.0607 & 0.0872 & 0.0888 \\
\hline 7 & \multirow{2}{*}{$\begin{array}{l}\text { Stator winding } \\
\text { interturn short } \\
\text { circuit }\end{array}$} & 0.0669 & 0.0894 & 0.1984 & 0.0769 & 0.3617 & 0.1368 & 0.0627 & 0.0072 \\
\hline 8 & & 0.0662 & 0.0879 & 0.2025 & 0.0807 & 0.3485 & 0.1101 & 0.0598 & 0.0443 \\
\hline
\end{tabular}

It is known from Table 1 that the energy values of each frequency band of the vibration signal in the normal condition but in different states are distinctly different. When a fault occurs in the wind turbine, the frequency component of the signal will change, and the energy value of the frequency band will change. In the process of fault, the frequency distribution information of different frequency bands can be expressed by the energy of each terminal point decomposed and reconstructed by wavelet packet.

\section{Fault Type Identification}

At present, the most popular method of intelligent fault diagnosis is supervised learning based pattern recognition, such as BP neural network, support vector machine and so on. These methods require a large number of samples of known fault categories to be pre trained to form classifiers, and then to identify unknown categories of samples. As shown in Fig. 5, support vector machines are used to classify and recognize the extracted feature signals.

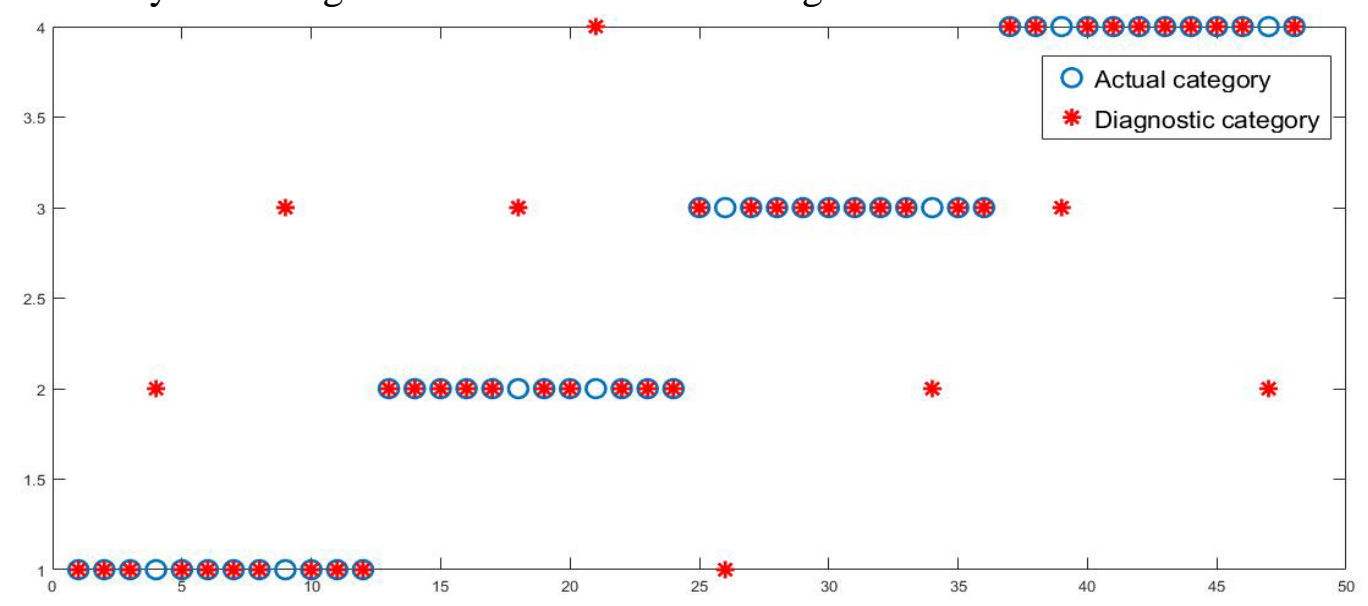

Fig. 5 Comparison between actual categories and diagnostic categories

\section{Conclusions}

This paper expounds two major problems in wind power generation. One is that the output of the wind turbine is fluctuating, which will cause the fluctuation of the voltage and frequency of the power grid and influence the power quality of the power grid. The other is that the working environment of the wind turbine is abominable, and many kinds of faults are easy to occur, causing the unit to stop and reduce the utilization rate of the unit. . Then, an example is given to illustrate the use of artificial intelligence technology in fault diagnosis of wind turbines. Artificial intelligence, as a new technology in the field of computer, has played a great role in solving the problems encountered in wind power generation. The powerful ability of artificial intelligence to deal with nonlinearity has great potential in solving the problems encountered in the development and utilization of new energy. 


\section{Acknowledgement}

This research was financially supported by National Natural Science Foundation of China (Grant No.51667018).

\section{References}

[1] Li Jingming, Wang Hongyan, Zhao Qun, Potential and Prospect of China's new energy resources, J. natural gas industry. 2008, 28 (1):149-153.

[2] Deng Wei, Liu Fangming, Jin Hai, Li Dan, New energy applications in cloud computing data center: research status and trends, J. Journal of computer science. 2013,36 (03): 582-598.

[3] Liao Zhiwei, Sun Yaming, Ye Qinghua, Application of artificial intelligence technology in power system fault diagnosis, J. Journal of power systems and automation. 2003 (06): 71-79.

[4] Wu Kaifeng, Liu Wantao, Li Yanhu, Based on cloud computing power large data analysis technology and application, J. China electric power. 2015, 48 (02): 111-116+127.

[5] Sun Hui, Xu Jian, Sun Yuanzhang, Yi Xiankun, Wind Farms Active Optimization Scheduling Based on Mixed Integer Linear Programming, J. Automation of Electric Power Systems. 2016, 40(22):27-33+42.

[6] Jiang Cheng, Liu Xianzheng, Probabilistic Evaluation of Wind Turbine Output, J. Modern Electric Power. 2013,30(01):51-54.

[7] Wang Zhongsheng, Intelligent Fault Diagnosis and Fault Tolerant Control, Northwestern Polytechnical University Press, 2005.

Reference to a chapter in an edited book: Chapter 8 Neural Network Fault Diagnosis and Fault Tolerant Control

[8] P.J. Tavner, J. Xiang, F. Spinato. Reliability analysis for wind turbines, J. Wind Energy. 2007, 10:1-18.

[9] Li Jingming, Wang Hongyan, Zhao Qun, Potential and prospects on new energy sources in China, J. Natural Gas Industry. 2008, 28(1):149-153.

[10] Liu W, Wang Z, Han J, Wind turbine fault diagnosis method based ondiagonal spectrum and clustering binary tree SVM, J. Renewable Energy. 2013, 50(3):1-6. 